\title{
Circuit
}

Musiques contemporaines

\section{Fragile Fixe Fugace : découvrir Denis Gougeon}

\section{Jonathan Goldman}

Volume 24, numéro 1, 2014

Denis Gougeon en six thèmes

URI : https://id.erudit.org/iderudit/1023646ar

DOI : https://doi.org/10.7202/1023646ar

Aller au sommaire du numéro

Éditeur(s)

Les Presses de l’Université de Montréal

ISSN

1183-1693 (imprimé)

1488-9692 (numérique)

Découvrir la revue

Citer ce document

Goldman, J. (2014). Fragile Fixe Fugace : découvrir Denis Gougeon. Circuit, 24(1), 4-7. https://doi.org/10.7202/1023646ar d'utilisation que vous pouvez consulter en ligne.

https://apropos.erudit.org/fr/usagers/politique-dutilisation/ 


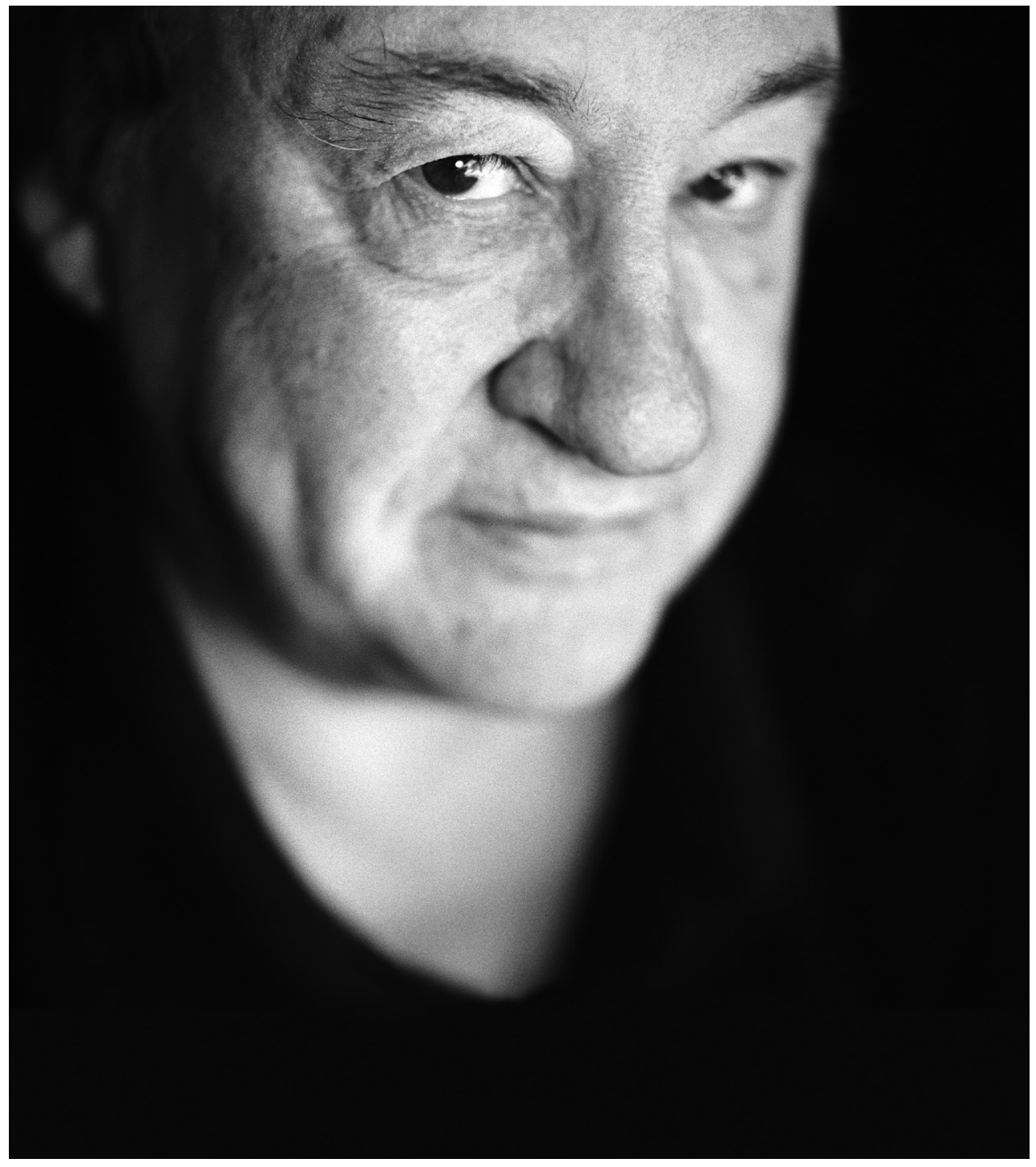

Denis Gougeon, 2013, par Florence Mennessier pour la SMCQ. 


\section{Fragile Fixe Fugace ${ }^{1}$ : découvrir Denis Gougeon}

Jonathan Goldman

On connait la formule: tous les deux ans depuis 2008, la Société de musique contemporaine du Québec (SMCQ) concentre sa saison artistique sur l'œuvre d'un seul compositeur. Dans le cadre de cette Série hommage, la SMCQ et son directeur artistique, Walter Boudreau, invitent des dizaines d'organismes de musique contemporaine à s'unir à elle pour célébrer la production de ce créateur. On se souvient encore de l'année Claude Vivier, premier compositeur «hommagé » en 2008, suivi, deux ans plus tard, par son maître Gilles Tremblay et, pour la saison 2011-2012, par la compositrice Ana Sokolović.

Nous voici donc, pour cette saison de la SMCQ (la $48^{8}$ !), à nouveau en pleine Série hommage, dédiée cette année à Denis Gougeon, compositeur aux multiples facettes, dont les quelque 50 événements organisés par la SMCQ nous permettent de savourer la production multiforme. Ajoutons qu'en attirant des spectateurs par milliers, ces événements mènent en même temps un combat contre la fragmentation croissante du milieu musical contemporain actuel. Mais la vocation de la Série hommage n'est pas seulement diffuser l'œuvre: elle participe activement à l'élargissement du catalogue du compositeur célébré par le moyen de la commande. Au cours de cette saison 2013-2014, plusieurs nouvelles créations de Denis Gougeon viendront enrichir son catalogue qui compte plus de 100 opus. Vous pourrez le constater en feuilletant, dans ce numéro, la liste des œuvres de Gougeon préparée par Solenn Hellégouarch.

Entièrement solidaire de la Série hommage, la revue Circuit consacre systématiquement un numéro au créateur célébré par la SMCQ. Rappelons que le vol. 18, nº 3 (2008) a été dédié à Claude Vivier, le vol. 20, nº 3 (2010) à Gilles Tremblay, et le vol. 22, n ${ }^{\circ} 3$ (2012) à Ana Sokolović. Ainsi, vous l'aurez deviné, le numéro actuel est consacré à l'œuvre de Denis Gougeon et s'inscrit
1. Titre d'une œuvre pour grand orchestre de Denis Gougeon commandée par l'Orchestre symphonique de Montréal, composée en 1991 et créée en 1993. 
2. Denis Gougeon (1996), "Garant communicateur, ou la parole agissante", Circuit, musiques contemporaines, vol. $7, \mathrm{n}^{\circ} 2$, p. 27-36

3. Voir: <www.musiccentre.ca/ centrestreams/swf ?mode=play by\&opt $=$ composer\&id $=356>$ (consulté le 6 janvier 2014).

4. On songe notamment à la sublime Clere Vénus (2001), sur des sonnets de Louise Labé, mais aussi à Éternité (1985) ou Maouna (1992). dans le cadre de la Série hommage dédiée à ce grand compositeur originaire de Granby.

La revue Circuit profite ainsi de l'occasion pour remédier à une présence encore relativement - et étrangement! - discrète du compositeur dans ses précédents numéros (si nous ne comptons pas un article de sa plume au sujet de son maître, Serge Garant, paru dans le vol. $7, \mathrm{n}^{\circ}$ 2, il y a déjà 17 ans de cela ${ }^{2}$ ). Me croirez-vous si je vous disais que Circuit avait déjà l'intention de consacrer un numéro à la musique de Gougeon bien avant que la SMCQ annonce l'identité de l'artiste «hommagé » en 2013-2014? Denis Gougeon jouit d'une carrière des plus impressionnantes en matière de création contemporaine au Québec: son œuvre aussi considérable qu'incontournable récompense une écoute attentive; un tel legs musical en devenir se fête et se doit d'être connu.

Une des particularités de la musique de Denis Gougeon se trouve sans doute dans son accessibilité sans tomber dans la complaisance. Sa musique toute sauf rébarbative - se fait invitation aux auditeurs et leur fait vivre ensuite une expérience qui a le potentiel d'étendre leurs horizons. Les pièces virtuoses de Gougeon pour instruments solo comme L'oiseau blessé (1987), pour flûte, et Piano-Soleil (1990), pour piano, sont jouées régulièrement dans les salles de concert et les conservatoires du monde entier. La qualité de son œuvre à travers la planète a par ailleurs été récemment soulignée lorsque Toy (Music Box), pour orchestre, a remporté le premier prix du Concours international de composition du Festival Présences à Shanghai (une coproduction francochinoise) en 2010. Cette œuvre est par ailleurs explorée dans ce numéro, en guise de Cahier d'analyse, par François-Hugues Leclair. Ajoutons en passant que sa musique est accessible au sens propre du terme: non moins de 32 enregistrements, y compris plusieurs interprétations d'une seule œuvre, peuvent être écoutés sans frais sur l'indispensable site du Centre de musique canadienne $(\mathrm{CMC})^{3}$. Cet outil gratuit devrait être considéré comme une annexe essentielle à ce numéro.

Mais l'œuvre de Gougeon fait surtout preuve d'une versatilité étourdissante. Le compositeur québécois a produit une grande quantité de musique instrumentale, bien sûr, mais l'on notera particulièrement un penchant certain pour la présence de la voix. Pensons à Heureux qui, comme... (1987), œuvre pour soprano et ensemble, écrite, comme tant d'autres ${ }^{4}$, pour son épouse Marie-Danielle Parent, la mythique soprano, qui a notamment créé Lonely Child (1981) de Vivier. Le compositeur fait également preuve d'un intérêt soutenu pour la musique de scène. Loin d'être de simples contrats «alimentaires» réalisés par un compositeur qui a su survivre au cours de longues années comme musicien freelance, ses musiques pour le théâtre, 
en particulier celles composées pour dix des créations de Denis Marleau, fondateur de la compagnie de création théâtrale UBU, se trouvent au cœur même de sa production et de ce que Denis Gougeon cherche à exprimer en tant qu'artiste. Comme la collaboration joue un rôle aussi primordial dans la démarche du compositeur, nous avons invité Denis Marleau à rendre hommage à son collaborateur de longue date en décrivant la démarche conjointe à laquelle ils se livrent dans la conception d'une œuvre scénique.

Bien que consacré à un créateur, ce nouveau numéro n’est pas conçu comme un simple hommage à Denis Gougeon : l'organe de réflexion qu'est Circuit vous propose une exploration de son univers musical afin de mieux apprécier le créateur et son œuvre. Dans cette optique, le numéro débute par un entretien par Françoise Davoine.

Denis Gougeon a intégré la Faculté de musique de l'Université de Montréal en 2001. Il a ainsi pu former deux ou trois cohortes de jeunes compositeurs. Lors d'un événement organisé le 8 novembre 2013 dans le cadre de la Série hommage, sept de ses anciens élèves (Ashot Ariyan, Simon Bertrand, André Cayer, Mathieu Lavoie, Analía Lludgar, Pierre Michaud et Marianne Trudel) ont composé des companion pieces aux Six thèmes solaires (1990) de leur professeur. L’enquête de ce numéro, menée par Maxime McKinley, interroge ces sept compositeurs sur leurs œuvres-hommages.

Enfin, les illustrations du numéro sont l'œuvre d'un artiste que Denis Gougeon côtoie depuis de longues années: le plasticien Michel Goulet, que l'on connaît bien au Québec grâce à ses nombreuses sculptures sur la place publique - on songe notamment à ces chaises iconiques qui ornent le belvédère du parc La Fontaine à Montréal. Ayant collaboré avec le sculpteur sur certaines créations de Denis Marleau depuis ${ }_{1993}$ (Roberto Zucco et Nathan le sage, dont une esquisse de la scénographie orne la couverture), Gougeon a trouvé, tout comme nous, qu'il serait fort approprié d'orner ce numéro «Gougeon » d'images d'œuvres de son ami Goulet.

Pour conclure ce numéro, vous trouverez dans la rubrique Actualités un compte rendu du livre À la lumière des mathématiques et à l'ombre de la philosophie: dix ans de séminaires mamuphi (dirigé par Moreno Andreatta, François Nicolas et Charles Alunni), préparé par Mathieu Bélanger, suivi d'une nouvelle chronique proposée par l'équipe du site Internet Cette ville étrange et rédigée par Paul Bazin. La rubrique se termine par nos traditionnelles nouveautés en bref concoctées par Isaiah Ceccarelli.

Bonne lecture! 